\title{
The fate of 'particles' in quantum field theories \\ with interactions
}

Doreen Fraser

Dept. of Philosophy

University of Waterloo

dlfraser@uwaterloo.ca

\begin{abstract}
Most philosophical discussion of the particle concept that is afforded by quantum field theory has focused on free systems. This paper is devoted to a systematic investigation of whether the particle concept for free systems can be extended to interacting systems. The possible methods of accomplishing this are considered and all are found unsatisfactory. Therefore, an interacting system cannot be interpreted in terms of particles. As a consequence, quantum field theory does not support the inclusion of particles in our ontology. In contrast to much of the recent discussion on the particle concept derived from quantum field theory, this argument does not rely on the assumption that a particulate entity be localizable.
\end{abstract}

KEYWORDS: quantum field theory, quanta, particle, interaction

\section{Introduction}

Quantum field theory (QFT) is the basis of the branch of physics known as "particle physics." However, the philosophical question of whether quantum field theories genuinely describe particles is not straightforward to answer. Since QFT's are formulated in terms of fields (i.e., mathematical expressions that associate quantities with points of spacetime), the issue is whether the formalism can be interpreted in terms of a particle notion. What is at stake is whether QFT, one of our current best physical theories, supports the inclusion of particles in our ontology. This 
paper advances an argument that, because systems which interact cannot be given a particle interpretation, QFT does not describe particles.

Even proponents of a particle interpretation of QFT acknowledge that the particle concept inherent in a QFT would differ from the classical particle concept in many ways. To distinguish the QFT concept from the classical one, the former has been dubbed the 'quanta' concept (e.g., Teller 1995, p. 29). Redhead and Teller (1992) argue that one way in which quanta differ from classical particles is that quanta are not capable of bearing labels. ${ }^{1}$ That is, they lack a property that is variously termed "haecceity," "primitive thisness," or "transcendental individuality." However, Teller argues that the quanta notion should still be considered a particlelike notion because quanta are aggregable (Teller 1995, p. 30). There are, for example, states in which we definitely have two quanta and states in which we definitely have three quanta, and these can be combined to yield a state in which we definitely have five quanta. Quanta are also particlelike insofar as they possess the same energies as classical, relativistic, non-interacting particles.

This minimal notion of quanta as entities satisfying a countability condition and a relativistic energy condition will be employed in the following investigation. These quanta may significantly differ from classical particles in other respects. For example, the question of whether there is an appropriate sense in which quanta are localized has been the subject of recent debate (see Malament (1996), Halvorson and Clifton (2002), and Fleming (2001)). However, for our purposes, this debate can be set aside. The particlelikeness of the quanta notion will not challenged; instead, the arguments presented below aim to show that the domain of application of the quanta concept is so strictly limited that quanta cannot be admitted into our ontology.

The quanta interpretation of QFT is based on special properties of the mathematical representation for free systems in QFT. Free fields describe the world in the absence of interactions. But in the real world there are always interactions. This raises a crucial question: can the quanta interpretation be extended to interacting systems? The analysis presented here aims to supply a comprehensive answer to this question. Ultimately, the answer is "no;" an interacting system

\footnotetext{
${ }^{1}$ For a dissenting view on the metaphysics of classical particles, see Huggett (1999).
} 
cannot be described in terms of quanta. This inquiry is in the same spirit as recent discussions (which are restricted to free systems) of whether a unique quanta notion is available for accelerating observers or in more general spacetime settings (i.e., nonstationary spacetimes) (Clifton and Halvorson 2001, Arageorgis et. al. 2003; Arageorgis et. al. 2002). The present investigation adopts the opposite approach: the restriction to free systems will be dropped, and the restriction to inertial observers on flat Minkowski spacetime will be retained. The commonality is that the interpretive conclusions rest on the employment of unitarily inequivalent representations of the canonical commutation relations. In this case, the fact that the representations for free and interacting systems are necessarily unitarily inequivalent is invoked in the first stage of the argument. However, the structure of the argument diverges from the above-mentioned discussions after this first stage; further work is required to establish that the unitarily inequivalent representation for the interacting field cannot possess the relevant formal properties.

After a brief review of the Fock representation for a free system and the standard argument that it supports a quanta interpretation, three methods for obtaining a quanta interpretation for an interacting system will be evaluated. The first method is simply to use the Fock representation for a free system to represent an interacting system. Since this method proves unsuccessful, it is necessary to generalize the definition of Fock representation so that it is applicable to interacting systems. In order to distinguish these definitions, I will reserve the term "Fock representation" for free systems and refer to the results of attempts to formulate analogous representations for interacting systems as " $\Phi O K$ representations." ${ }^{2}$ In principle, there are two methods for extending the definition of a Fock representation which are allied with the two approaches to defining a Hilbert space representation in quantum field theory: the 'constructive' method of applying to an interacting field the same quantization procedure that generates a Fock representation from a classical free field and the 'axiomatic' method of specifying a Hilbert space representation by stipulating formal conditions. The former method will be investigated in Section 4 and the latter in Section 5. Following the failure of both methods, a final, last-ditch attempt to retain a quanta

\footnotetext{
${ }^{2}$ This expression is borrowed from the early history of QFT, when what is here referred to as a "Fock representation" was sometimes labeled a " $\Phi O K$ representation."
} 
interpretation for interacting systems will be critiqued in Section 6. The implications of the conclusion that interacting systems cannot be described in terms of quanta for metaphysics and for the foundations of QFT will be assessed in Section 7.

\section{The Fock representation for a free field}

Every introductory QFT textbook contains a discussion of how to construct a Fock space representation of the equal-time canonical commutation relations (ETCCR's) for a free field. The construction proceeds by effecting a positive-negative Fourier decomposition of a classical free field and then promoting the coefficients to operators. The details of this construction will be discussed in Section 4. In this section, the properties of the final product of the construction-a Fock representation for a free field-will be reviewed. Strictly speaking, the textbook characterization of a Fock representation is not well-defined. This can be remedied by making a few modifications. However, for the purposes of this section, the textbook treatment will be used because the argument that a Fock representation supports a quanta interpretation is most naturally formulated using the unrigorous presentation. Details of the rigorous version can be found in the footnotes.

A Fock representation for a free bosonic real scalar field with $m>0$ on Minkowski spacetime ${ }^{3}$ possesses the following formal properties: ${ }^{4}$

1. Field operators There exist well-defined annihilation and creation operators $a(\mathbf{k}, t), a^{\dagger}(\mathbf{k}, t)$

\footnotetext{
${ }^{3}$ The free bosonic real scalar field is treated for simplicity; other types of free fields also possess Fock representations. The restriction to $m>0$ and (flat) Minkowski spacetime ensures that there exists a Fock representation that is unique up to unitary equivalence and supports a quanta interpretation in the manner described below. For accounts of what happens in other spacetime settings see Wald $(1994, \S \S 4.3,4.4)$ and Arageorgis, Earman \& Ruetsche (2002).

${ }^{4} \mathrm{~A}$ note on how to make these properties rigorously well-defined. The consequence of allowing the integrals to range over all of space is that the $n$-particle states are not normalizable. In addition, the integral in equation (2) does not converge (Wald 1994, p. 35). To fix the first problem, it is often stipulated that $\phi(\mathbf{x}, t)$ is confined to a box or satisfies periodic boundary conditions (see, e.g., Roman (1969, p. 50)), but this is not necessary. Both problems can be resolved by 'smearing' the fields in their spatial variables. See the definition of a $\Phi O K_{2}$ representation in Section 5 .
} 
where $\mathbf{k}^{2}=k_{0}^{2}-m^{2} . a(\mathbf{k}, t), a^{\dagger}(\mathbf{k}, t)$ obey the ETCCR's

$$
\left[a(\mathbf{k}, t), a\left(\mathbf{k}^{\prime}, t\right)\right]=0,\left[a^{\dagger}(\mathbf{k}, t), a^{\dagger}\left(\mathbf{k}^{\prime}, t\right)\right]=0,\left[a(\mathbf{k}, t), a^{\dagger}\left(\mathbf{k}^{\prime}, t\right)\right]=\delta^{3}\left(\mathbf{k}-\mathbf{k}^{\prime}\right)
$$

At any given time $t$ the quantum field $\phi(\mathbf{x}, t)$ can be defined as follows ( where $\omega_{\mathbf{k}}^{2}=k_{0}^{2}=$ $\left.\mathbf{k}^{2}+m^{2}\right)$

$$
\phi(\mathbf{x}, t)=\int \frac{d^{3} k}{(2 \pi)^{\frac{3}{2}} \sqrt{2 \omega_{\mathbf{k}}}}\left[a^{\dagger}(\mathbf{k}, t) e^{i k \cdot x}+a(\mathbf{k}, t) e^{-i k \cdot x}\right]
$$

The conjugate momentum field $\pi(\mathbf{x}, t)$ is defined using $\pi(\mathbf{x}, t)=\frac{\partial \phi(\mathbf{x}, t)}{\partial t}$ and $\frac{\partial a(\mathbf{k}, t)}{\partial t}=$ $\frac{\partial a^{\dagger}(\mathbf{k}, t)}{\partial t}=0$ :

$$
\pi(\mathbf{x}, t)=\int \frac{d^{3} k}{(2 \pi)^{\frac{3}{2}} \sqrt{2 \omega_{\mathbf{k}}}}\left[i \omega_{\mathbf{k}} a^{\dagger}(\mathbf{k}, t) e^{i k \cdot x}-i \omega_{\mathbf{k}} a(\mathbf{k}, t) e^{-i k \cdot x}\right]
$$

Inverting and solving for $a(\mathbf{k}, t), a^{\dagger}(\mathbf{k}, t)$ gives

$$
\begin{aligned}
a(\mathbf{k}, t) & =\int \frac{d^{3} x}{(2 \pi)^{\frac{3}{2}} \sqrt{2 \omega_{\mathbf{k}}}} e^{i k \cdot x}\left[\omega_{\mathbf{k}} \phi(\mathbf{x}, t)+i \pi(\mathbf{x}, t)\right] \\
a^{\dagger}(\mathbf{k}, t) & =\int \frac{d^{3} x}{(2 \pi)^{\frac{3}{2}} \sqrt{2 \omega_{\mathbf{k}}}} e^{i k \cdot x}\left[\omega_{\mathbf{k}} \phi(\mathbf{x}, t)-i \pi(\mathbf{x}, t)\right]
\end{aligned}
$$

2. No-particle state There exists a unique (up to phase factor) 'no-particle state' $|0\rangle$ such that

$$
a(\mathbf{k}, t)|0\rangle=0 \text { for all } \mathbf{k}
$$

3. Number operators Number operators $N(\mathbf{k})$ can be defined for any $t$ :

$$
\begin{aligned}
N(\mathbf{k}) & =a^{\dagger}(\mathbf{k}, t) a(\mathbf{k}, t) \\
N(\mathbf{k})\left[a^{\dagger}(\mathbf{k}, t)^{n}|0\rangle\right] & =n\left[a^{\dagger}(\mathbf{k}, t)^{n}|0\rangle\right]
\end{aligned}
$$

where $n=\{0,1,2, \ldots\}^{5}$

\footnotetext{
${ }^{5}$ When normalized, the $n$-particle state becomes $\frac{a^{\dagger}(\mathbf{k}, t)^{n}|0\rangle}{\sqrt{n !}}$.
} 
In addition, for any $t$, the total number operator $N=\int d^{3} k N(\mathbf{k})=\int d^{3} k a^{\dagger}(\mathbf{k}, t) a(\mathbf{k}, t)$ is well-defined. ${ }^{6}$

4. Fock space The one-particle Hilbert space $\mathcal{H}$ has as a basis the set of vectors generated from $|0\rangle$ by single applications of $a^{\dagger}(\mathbf{k}, t)$ (for any $\mathbf{k}$ satisfying $\mathbf{k}^{2}=k_{0}^{2}-m^{2}$ ). The Fock space $\mathcal{F}$ for $\phi(\mathbf{x}, t)$ is obtained by taking the direct sum of the $n$-fold symmetric tensor product of $\mathcal{H}: \mathcal{F}(\mathcal{H})=\oplus_{n=0}^{\infty}\left(\otimes^{n} \mathcal{H}\right)($ Wald 1994, p. 192). $|0\rangle$ is cyclic with respect to the $a^{\dagger}(\mathbf{k}, t)^{\prime} \mathbf{s}$.

Before turning to the quanta interpretation, note that this set of formal properties prescribes a physically appropriate representation for a free system. $a(\mathbf{k}, t), a^{\dagger}(\mathbf{k}, t), N(\mathbf{k})$, and $N$ are relativistically covariant, as is required of physically significant quantities in a relativistic theory like QFT. Also, this set of formal properties uniquely characterizes a Fock representation. As a consequence of the failure of the Stone-von Neumann theorem for an infinite number of degrees of freedom, ${ }^{7}$ there are uncountably many unitarily inequivalent ${ }^{8}$ representations of the ETCCR's. ${ }^{9}$

\footnotetext{
${ }^{6}$ That is, when the number operators are properly defined using a test function space $\mathcal{T}$, as in Section 5 , $N=\sum_{j=1}^{\infty} a^{\dagger}\left(f_{j}\right) a\left(f_{j}\right)$ converges in the sense of strong convergence on the domain of $N$ where $\left\{f_{j}\right\}$ is an orthonormal basis of $\mathcal{T}$ and $N$ exists only if $N$ exists and is the same for every choice of orthonormal basis $\left\{f_{j}\right\}$ (Dell'Antonio, Doplicher \& Ruelle (1966), pp. 225-226).

${ }^{7}$ See Ruetsche (2002) for an exposition.

${ }^{8}$ Two representations of the ETCCR's $\left(\mathcal{H}_{1},\left\{O_{1}^{i}\right\}\right)$ and $\left(\mathcal{H}_{2},\left\{O_{2}^{i}\right\}\right)$ (where $\left\{O_{n}^{i}\right\}$ is the collection of operators appearing in the ETCCR's) are unitarily equivalent if and only if there exists some unitary mapping $U$ from Hilbert space $\mathcal{H}_{1}$ to Hilbert space $\mathcal{H}_{2}$ that such that for each operator $O_{1}^{j} \in\left\{O_{1}^{i}\right\}$ there exists an operator $O_{2}^{j}=U O_{1}^{j} U^{-1} \in\left\{O_{2}^{i}\right\}$ (Wald 1994, p. 19). The practical importance of unitary equivalence is that-assuming $\left\{O_{1}^{i}\right\}$ and $\left\{O_{2}^{i}\right\}$ include all physically significant operators-if two representations are unitarily equivalent then they will be physically equivalent in the sense that both produce the same expectation values for all physically significant operators. See Ruetsche (2002) for further discussion.

${ }^{9} \mathrm{~A}$ representation of the Weyl form of the ETCCR's is given by the pair of families of unitary operators $U(f, t)$, $V(g, t)(f, g \in \mathcal{T})$ in the usual way, where $U(f, t)=e^{i \phi(f, t)}, V(g, t)=e^{i \pi(g, t)}$ and $U(f, t)$ and $U(g, t)$ satisfy the usual conditions (Wightman 1967b, p. 189)

$$
\begin{aligned}
& U(f, t) U(g, t)=U(f+g, t) \\
& V(f, t) V(g, t)=V(f+g, t)
\end{aligned}
$$$$
U(f, t) V(g, t)=e^{i(f, g)} V(g, t) U(f, t)
$$

and $U(\alpha f, t), V(\alpha g, t)$ are continuous in the real number $\alpha$. The Weyl form of the ETCCR's is more convenient than the standard form$$
[\phi(f, t), \phi(g, t)]=[\pi(f, t), \pi(g, t)]=0,[\phi(f, t), \pi(g, t)]=i(f, g)
$$

because, unlike $\phi(f, t)$ and $\pi(f, t)$, the exponentiated operators $U(f, t)$ and $V(f, t)$ are bounded, so they are
} 
It has been proven that (when suitably rigorized), these properties pick out an irreducible representation of the ETCCR's that is unique up to unitary equivalence (Gårding and Wightman 1954, pp. 624-625; Wightman and Schweber 1955, pp. 819-822). More precisely, if $\phi_{m}$ is the free field on Minkowski spacetime satisfying the Klein-Gordon equation with mass $m$, then the representation of the ETCCR's for $\phi_{m}(\mathbf{x}, t), \pi_{m}(\mathbf{x}, t)$ that possesses properties (1) to (4) is unique (up to unitary equivalence). However, it should also be noted that the Fock representation of the ETCCR's for the field $\phi_{m^{\prime}}(\mathbf{x}, t), m^{\prime} \neq m$, is unitarily inequivalent to the Fock representation of the ETCCR's for $\phi_{m}(\mathbf{x}, t)$ (Reed \& Simon 1975, pp. 233-235). Therefore, there is such a thing as the Fock representation for a free field that satisfies a specified Klein-Gordon equation; there are a multitude of unitarily inequivalent Fock representations when the field equation is left unspecified.

The existence of a well-defined operator $N$ with a spectrum consisting of the non-negative integers is not in itself sufficient for a quanta interpretation. Quantum theories contain discrete properties. How, then, do we know that $N$ is counting entities rather than, say, energy levels? An argument needs to be made that $N$ counts quanta, i.e., $|0\rangle$ is a state in which there are no quanta, $a^{\dagger}(\mathbf{k}, t)|0\rangle$ is a state in which there is one quantum, etc. A Fock representation supports a quanta interpretation because the eigenvectors of $N$, the total number operator, possess properties that are appropriate for states containing definite numbers of particles. These properties arise from all of the continuous and discrete symmetries of the field equation, but, for the purposes of the ensuing discussion, we will restrict our attention to time translations and energy. In the Fock automatically defined on the same domain. Of course, 'unsmearing' this form of the ETCCR's yields the familiar relations

$$
\left[\phi(\mathbf{x}, t), \phi\left(\mathbf{x}^{\prime}, t\right)\right]=0,\left[\pi(\mathbf{x}, t), \pi\left(\mathbf{x}^{\prime}, t\right)\right]=0,\left[\phi(\mathbf{x}, t), \pi\left(\mathbf{x}^{\prime}, t\right)\right]=i \delta^{3}\left(\mathbf{x}-\mathbf{x}^{\prime}\right)
$$

It may turn out that the ETCCR's do not hold for physically realistic interactions because the arguments of the field operators must be smeared in time as well as space (see Streater and Wightman 2000, p. 168). However, this potential complication will be overlooked here. Note that this is not a universal feature of interacting theories; there are rigorous Hilbert space models for non-trivial interactions in which the ETCCR's do hold (e.g., for a Lagrangian with a scalar $\phi^{4}$ interaction term in two spacetime dimensions) (Glimm and Jaffe 1970). 
representation, the Hamiltonian operator $H$ is (for any $t)^{10}$

$$
H=\int d^{3} k \omega_{\mathbf{k}} a^{\dagger}(\mathbf{k}, t) a(\mathbf{k}, t)=\int d^{3} k \omega_{\mathbf{k}} N(\mathbf{k})
$$

This expression for $H$ can be used to establish that the eigenvectors of $N$ have the correct energies for states in which the indicated number of quanta is present.

1. $|0\rangle$, the no-particle state It can easily be verified that $H|0\rangle=0$. Since the spectrum of $H$ does not contain any negative eigenvalues, ${ }^{11}$ this means that $|0\rangle$ is the ground state of $H$. Moreover, we can infer from the uniqueness of the no-particle state (property 2 above) that $|0\rangle$ is the unique ground state of $H$. Since the state in which there are no particles would presumably have the lowest energy, if $|0\rangle$ cannot be interpreted as the no-particle state, then there is no other candidate in a Fock representation.

Furthermore, $|0\rangle$ is invariant under time translations: $e^{i H t}|0\rangle=|0\rangle$. More generally, $|0\rangle$ is the unique state which carries the trivial, one-dimensional representation of all symmetry groups $^{12}$ (Roman 1969, p. 79). Consequently, $|0\rangle$ is the physical vacuum state. As Streater and Wightman remark, since $|0\rangle$ is invariant under the unitary operators that give a representation of the Poincaré group and we are operating in Minkowski spacetime, $|0\rangle$ "looks the same to all observers" (i.e., observers in inertial motion) (2000, p. 21). Nothing-i.e., the absence of particles-is something that we would expect to look the same to all inertial observers. Thus, it is reasonable to interpret $|0\rangle$ as a state in which there are no particles because it is the unique state with the desired properties: it is the lowest energy state and looks the same to all inertial observers.

2. $a^{\dagger}(\mathbf{k}, t)|0\rangle$ (where $\mathbf{k}^{2}=k_{0}^{2}-m^{2}$ ), the one-particle states The one-particle states furnish

\footnotetext{
${ }^{10}$ After normal ordering; i.e., moving all the $a^{\dagger}(\mathbf{k}, t)$ 's to the left of the $a(\mathbf{k}, t)$ 's without using the ETCCR's in (1).

${ }^{11}$ Because $a^{\dagger}(k)=0$ when $k_{0}<0$. For details, see the explication of the positive-negative frequency decomposition of $\psi(x)$ in Section 4.

${ }^{12}$ The Poincaré group and, when applicable, a symmetry group associated with charge and any discrete symmetry groups.
} 
the definitive argument for the quanta interpretation. States of the form $a^{\dagger}(\mathbf{k}, t)|0\rangle$ are also eigenstates of $H: H\left[a^{\dagger}(\mathbf{k}, t)|0\rangle\right]=\sqrt{\mathbf{k}^{2}+m^{2}}\left[a^{\dagger}(\mathbf{k}, t)|0\rangle\right]$. Special relativity dictates that $\sqrt{\mathbf{k}^{2}+m^{2}}$ is the correct energy for a single non-interacting particle with momentum $\mathbf{k}$ and mass $m$. This correspondence between the energy eigenvalues associated with these quantum states and the relativistic energies of single classical particles provides a justification for interpreting $a^{\dagger}(\mathbf{k}, t)|0\rangle$ as a state containing a single quantum of momentum $\mathbf{k}$ and mass $m .^{13}$

3. $a^{\dagger}(\mathbf{k}, t)^{n}|0\rangle \quad(n \geq 2)$, the $n$-particle states All of the other eigenvectors of $N(\mathbf{k})$ also have the correct relativistic energies for states in which there are particular numbers of non-interacting particles. In general, $H\left[a^{\dagger}(\mathbf{k}, t)^{n}|0\rangle\right]=n \omega_{\mathbf{k}}\left[a^{\dagger}(\mathbf{k}, t)^{n}|0\rangle\right]$ (where $\omega_{\mathbf{k}}=$ $\left.\sqrt{\mathbf{k}^{2}+m^{2}}\right)$, so $a^{\dagger}(\mathbf{k}, t)^{n}|0\rangle$ is an eigenstate of $H$ with the correct relativistic energy for a state in which there are $n$ non-interacting particles, each with momentum $\mathbf{k}$. This analysis generalizes to eigenvectors of the total number operator $N$ in a straightforward way:

$$
\begin{aligned}
H\left[a^{\dagger}\left(\mathbf{k}_{1}, t\right)^{n_{1}} a^{\dagger}\left(\mathbf{k}_{2}, t\right)^{n_{2}} \cdots a^{\dagger}\left(\mathbf{k}_{l}, t\right)^{n_{l}}|0\rangle\right] & = \\
& \left(n_{1} \omega_{\mathbf{k}_{1}}+n_{2} \omega_{\mathbf{k}_{2}}+\ldots+n_{l} \omega_{\mathbf{k}_{l}}\right)\left[a^{\dagger}\left(\mathbf{k}_{1}, t\right)^{n_{1}} a^{\dagger}\left(\mathbf{k}_{2}, t\right)^{n_{2}} \cdots a^{\dagger}\left(\mathbf{k}_{l}, t\right)^{n_{l}}|0\rangle\right]
\end{aligned}
$$

therefore, $a^{\dagger}\left(\mathbf{k}_{1}, t\right)^{n_{1}} a^{\dagger}\left(\mathbf{k}_{2}, t\right)^{n_{2}} \cdots a^{\dagger}\left(\mathbf{k}_{l}, t\right)^{n_{l}}|0\rangle$ has the correct relativistic energy for a state in which there are $n_{1}$ non-interacting particles with momentum $\mathbf{k}_{1}, n_{2}$ non-interacting particles with momentum $\mathbf{k}_{2}$, etc.

Thus the energy eigenvalues provide a physical justification for interpreting any $n$-particle eigenvector of $N$ as a state in which there are $n$ non-interacting quanta.

There is a formal analogy between the Fock representation for a free field and an infinite array

\footnotetext{
${ }^{13}$ This correspondence to relativistic energies holds for sharp values of $\mathbf{k}$. However, to make the characterization of the Fock representation for a free field rigorous, the momentum argument of $a^{\dagger}(\mathbf{k}, t)$ must be smeared: $a^{\dagger}(\tilde{f}, t)=$ $\int a^{\dagger}(\mathbf{k}, t) \tilde{f}(\mathbf{k}) d \mathbf{k}$ where $\tilde{f}$ is the Fourier transform of $f \in \mathcal{T}$ (see footnote 4 above). For this formulation, an argument could made that $\int \tilde{f}(\mathbf{k}) \sqrt{\mathbf{k}^{2}+m^{2}} d \mathbf{k}$ is the correct relativistic energy for a single particle with unsharp momentum distributed according to $\tilde{f}(\mathbf{k})$.
} 
of quantum mechanical simple harmonic oscillators (SHO's) (e.g., a quantum mass on a spring). This analogy does not do any philosophical work in this paper, but it is useful background because philosophers often couch their discussions of QFT in terms of this analogy. Associated with a single SHO are the "ladder operators" (or "lowering" and "raising" operators):

$$
\begin{aligned}
a & =x \sqrt{\frac{m \omega}{2}}+i p \sqrt{\frac{1}{2 m \omega}} \text { where } \omega^{2} \text { is the frequency of the SHO } \\
a^{\dagger} & =x \sqrt{\frac{m \omega}{2}}-i p \sqrt{\frac{1}{2 m \omega}}
\end{aligned}
$$

Note the formal similarity between $(9 \mathrm{a}, 9 \mathrm{~b})$ and $a(\mathbf{x}, t), a^{\dagger}(\mathbf{x}, t)$ (the Fourier transforms of (4a, 4b)). Written in terms of $a, a^{\dagger}$, the Hamiltonian for a SHO is

$$
H=\frac{1}{2} \varpi\left(a^{\dagger} a+a a^{\dagger}\right)
$$

Prior to normal ordering and when the system is placed in a box, the Hamiltonian for a free quantum field is an infinite sum of SHO Hamiltonians of different frequencies:

$$
H=\sum_{k} \frac{\omega_{\mathbf{k}}}{2}\left[a^{\dagger}(\mathbf{k}, t) a(\mathbf{k}, t)+a(\mathbf{k}, t) a^{\dagger}(\mathbf{k}, t)\right]
$$

In this manner, the Fock representation for a free system can sustain a quanta interpretation. But is it also the case that an interacting system possesses a Hilbert space representation that admits a quanta interpretation? In the next section, the possibility of using the Fock representation for a free system to represent an interacting system will be ruled out. This leaves the alternative strategy of attempting to generalize the definition of a Fock representation for a free system so that it can also be applied to an interacting system. Two approaches fall into this category: focusing on the motivation behind the relationship between $\phi(\mathbf{x}, t), \pi(\mathbf{x}, t)$ and $a(\mathbf{k}, t)$, $a^{\dagger}(\mathbf{k}, t)$ expressed in identities (2), (3) and focusing on the four formal properties of the free Fock representation listed above. The motivation for the expressing $\phi(\mathbf{x}, t)$ in terms of $a(\mathbf{k}, t), a^{\dagger}(\mathbf{k}, t)$ in (2) is that this is the output of the quantization procedure that generates operator expressions 
for the free quantum fields from the free classical fields. The relevant step in the quantization procedure is the positive-negative frequency decomposition of the classical field. One approach to extending the definition of Fock representation to interacting fields is to apply the same quantization procedure to an interacting field (i.e., to decompose the classical interacting field into positive and negative frequency parts). To avoid confusing the representation that results with the Fock representation for a free field, the former will be labelled a "ФOK $K_{1}$ representation." This approach will be investigated in Section 4; as we shall see, it is not feasible.

The second approach to extending the definition of Fock representation to interacting systems is to focus attention on the formal properties of a Fock representation listed above, without regard for their origins. Instead of defining a representation by quantizing a classical field, a unique (up to unitary equivalence) representation of the ETCCR's is picked out by stipulating formal properties. As noted, the four formal properties listed above do pick out a unique (up to unitary equivalence) representation of the ETCCR's for a given set of canonical free fields $\left(\phi_{F}, \pi_{F}\right)$. If the same set of properties could be used to pick out a unique representation of the ETCCR's for a given set of canonical interacting fields $\left(\phi_{I}, \pi_{I}\right)$, then the representation singled out in this way could be considered the 'Fock' representation for this interacting field. Work by Wightman and his collaborators provides a starting point for extending the definition of Fock representation to interacting fields in this way. Such a representation will be dubbed a " $\Phi O K_{2}$ representation." The question of whether a $\Phi O K_{2}$ representation can sustain a quanta interpretation for an interacting system will be the subject of Section 5. Once again, the conclusion will be that a $\Phi O K_{2}$ representation fails to yield a quanta interpretation for an interacting field.

To motivate the search for an extension of the definition of Fock representation to interacting fields, the next section advances an argument based on Haag's theorem that a Fock representation for a free field cannot sustain a quanta interpretation for an interacting field. 


\section{Method \#1: Using the Fock representation for a free}

\section{field}

The simplest strategy for obtaining a quanta interpretation for an interacting field would be to use the Fock representation for a free system to represent a given interacting system, and then to try to extract a quanta interpretation. That is, the hope is that the Hilbert space spanned by $n$-particle states for the free field contains states that can be interpreted as containing $n$ quanta in the presence of the interaction. As we shall see, this simple strategy fails because there is no state in the Fock representation for a free field that can be interpreted as containing zero quanta.

The background to this argument is the existence of different approaches to QFT with interactions. "Canonical QFT" is the version of the theory that is found in most introductory QFT textbooks. It was developed by Feynman, Dyson, and their colleagues. Mathematical physicists concerned with the lack of mathematical rigor in canonical QFT adopted an axiomatic approach to the theory. Prominent contributors to this tradition include Wightman and Haag.

Haag's theorem pinpoints the source of the problem with the strategy of obtaining a quanta interpretation for an interacting system from the Fock representation for some free system. The consensus among axiomatic quantum field theorists is that Haag's theorem entails that a Fock representation for a free field cannot be used to represent an interacting field (e.g., Wightman (1967a, p.250; 1967b, p. 193; 1989, p. 610), Haag (1992, p. 55), Lopuszanski (1961, p. 747), Bratteli and Robinson (1996, p. 218), Bogolubov et al. (1975, p. 560); also Heathcote (1989, p. 91)). ${ }^{14}$ Translated into mathematical terms, the assumption that a given interacting field can be represented using the Fock representation for a free field amounts to the assumption that the Fock representation $\mathcal{F}$ of the ETCCR's for the canonical free fields $\left(\phi_{F}(x), \pi_{F}(x)\right)$ is unitarily equivalent to the Hilbert space representation $\mathcal{H}_{I}$ of the ETCCR's for the interacting

\footnotetext{
${ }^{14}$ For example, Wightman writes that "[a] necessary condition that a[n interacting] theory make physical sense is therefore that one use a strange representation of the commutation relations" (1967a, p. 250). A "strange representation" is any representation of the ETCCR's for a free field that is unitarily equivalent to the Fock representation (Bogolubov et. al. 1975, p. 560).
} 
fields $\left(\phi_{I}(x), \pi_{I}(x)\right)$ at all times:

For all $t$ there exists a unitary transformation $U(t): \mathcal{F} \rightarrow \mathcal{H}_{I}$ such that

$$
\phi_{I}(\mathbf{x}, t)=U(t) \phi_{F}(\mathbf{x}, t) U^{-1}(t) ; \quad \pi_{I}(\mathbf{x}, t)=U(t) \pi_{I}(\mathbf{x}, t) U^{-1}(t)
$$

This assumption underlies the interaction picture representation employed in canonical QFT. The interaction picture is undermined by Haag's theorem. In short, the theorem states that if all the assumptions of the interaction picture are accepted, then $\left(\phi_{I}(\mathbf{x}, t), \pi_{I}(\mathbf{x}, t)\right)$-the fields that were intended to describe the interaction-actually describe a free system. As Streater and Wightman put it, "the interaction picture exists only if there is no interaction" (2000, p. 166). (For an in-depth analysis of Haag's theorem see Earman and Fraser (2006)).

The version of Haag's theorem proven in Hall and Wightman (1957) establishes that, given certain physically reasonable assumptions, $U(t)$ does not exist at any time. That is, any irreducible Hilbert space representation of the ETCCR's for genuinely interacting fields $\left(\phi_{I}(x), \pi_{I}(x)\right)$ is unitarily inequivalent to a Fock representation for any free fields at all times. The physically reasonable premises of the theorem are as follows:

(i) No states of negative energy exist.

(ii) Poincaré transformations on $\mathcal{F}$ and $\mathcal{H}_{I}$ are represented by unitary operators $P_{i}$ $(i=F, I)$, respectively, which follows from Wigner's theorem concerning the representation of symmetries (Streater and Wightman 2000, pp. 7-8).

(iii) $\left(\phi_{i}(x), \pi_{i}(x)\right)$ are covariant under Poincaré transformations $P_{i}$, which is a prerequisite from relativity theory for $\left(\phi_{i}(x), \pi_{i}(x)\right)$ to be independent of inertial reference frame.

(iv) There exist unique normalizable vacuum states $\left|\Phi_{i}\right\rangle$ such that $P_{i}\left|\Phi_{i}\right\rangle=0$, which follows from Wigner's classification of representations of the Poincaré group (Streater and Wightman 2000, pp. 21-22). 
The final assumption is also underwritten by the quanta interpretation for a free field: the noparticle state $\left|0_{F}\right\rangle$ in a Fock representation coincides with the vacuum state $\left|\Phi_{F}\right\rangle$; the invariance of $\left|0_{F}\right\rangle$ under Poincaré transformations means that this state looks the same to all inertial observers.

What would happen if one paid no heed to the lesson of Haag's theorem and insisted on retaining the assumption that the Hilbert space representation for $\left(\phi_{I}, \pi_{I}\right)$ is unitarily equivalent to the Fock representation for $\left(\phi_{F}, \pi_{F}\right)$ ? This is the course taken by canonical quantum field theorists, who continue to employ the interaction picture. The answer suggested by Haag's theorem is that $H_{I}\left|\Phi_{I}\right\rangle=\infty$ where $H_{I}$ is the total interaction Hamiltonian. The implied expectation value $\left\langle\Phi_{I}\left|H_{I}\right| \Phi_{I}\right\rangle=\infty$ has been confirmed by non-perturbative calculations for bosonic $\left(\phi^{2 n}\right)_{s+1}$ $(n \geq 1)$ self-interaction terms in spacetime of dimension $(s+1) \geq 2$ (Glimm and Jaffe 1969). As a consequence, this representation cannot sustain a quanta interpretation for the interacting field. Recall that the justification for regarding $n$-particle states in a Fock representation for a free field as states in which a definite number of quanta are present is that these states possess the appropriate energy eigenvalues (see Section 2). The formal ${ }^{15}$ meaning of $H_{I}\left|\Phi_{I}\right\rangle=\infty$ is that $\left|\Phi_{I}\right\rangle$ is not in the domain of $H_{I}$. This means that $\left|\Phi_{I}\right\rangle$ is not associated with an energy expectation value, which undercuts the justification for regarding $\left|\Phi_{I}\right\rangle$ as a state in which there are no quanta. However, $\left|\Phi_{I}\right\rangle$ is the only state that is invariant under Poincaré transformations; since $\left|\Phi_{I}\right\rangle$ is the only state that looks the same to all inertial observers, it is the only candidate no quanta state. Therefore, there is no state in the interaction picture that, in the presence of the interaction, can reasonably be interpreted as a state in which no quanta are present, and the interaction picture does not support a quanta interpretation for the interacting field. Put in different terms, the Fock representation for a free field does not furnish a quanta interpretation for an interacting field.

In fact, the true situation is probably even worse than Haag's theorem indicates. Haag's theorem only pertains to the application of $H_{I}$ to the vacuum $\left|\Phi_{I}\right\rangle$. In general, it seems likely that interactions in four-dimensional spacetime are are such that $H_{I}\left|\Psi_{I}\right\rangle=\infty$ where $\left|\Psi_{I}\right\rangle=U(t)\left|\Psi_{F}\right\rangle$

\footnotetext{
${ }^{15} \mathrm{An}$ informal reading of $H_{I}\left|\Phi_{I}\right\rangle=\infty$ yields the same conclusion. Informally, the expression $H_{I}\left|\Phi_{I}\right\rangle=\infty$ indicates that $\left|\Phi_{I}\right\rangle$ is a state of infinite energy. Therefore, $\left|\Phi_{I}\right\rangle$ is not a physically possible state.
} 
and $\left|\Psi_{F}\right\rangle$ is any state in $\mathcal{F}$ (see Glimm (1969, p. 104)). This means that not only is there no zero quanta state in the interaction picture representation, but there are also no one, two, three, etc. quanta states.

Before considering proposals for generalizing the definition of Fock representation to interacting fields, I will respond to an obvious objection to this line of argument. Canonical quantum field theorists are aware that the interaction picture representation produces infinite expectation values for energy and they correct this problem by renormalizing the Hamiltonian $H_{I}$. For example, they incorporate an infinite counterterm $E$ into the renormalized Hamiltonian $H_{I}^{r e n}$ $\left(H_{I}^{r e n}=H_{I}-E\right)$ so that (at least informally) $H_{I}^{r e n}\left|\Phi_{I}\right\rangle=0$. Then, so the objection goes, there is no longer any obstacle to a quanta interpretation; $\left|\Phi_{I}\right\rangle$ is an eigenstate of $H_{I}^{\text {ren }}$ with the correct energy eigenvalue for a state in which there are no quanta.

A complete response to this objection would be lengthy and take me far outside the scope of this paper. ${ }^{16}$ The brief (but adequate) response is that $H_{I}^{r e n}$ is not a well-defined self-adjoint operator on the interaction picture representation in virtue of the fact that it contains an infinite term. This means that time translations are not represented in the usual way by the unitary operators $\exp \left(-i H_{I}^{r e n} t\right)$. I urge that, unless a good reason for abandoning this prima facie plausible assumption about how to formulate QFT presents itself, the assumption should be retained. Haag's theorem does not furnish a good reason for abandoning this assumption because the premise that free and interacting Hilbert space representations are unitarily equivalent is unjustified. The consequence of the failure of the Stone-von Neumann theorem in QFT is that there exist uncountably many unitarily inequivalent representations of the ETCCR's; there exist many possible Hilbert space representations for an interaction that are unitarily inequivalent to the Fock representation for any free field, and one of these may admit a well-defined self-adjoint Hamiltonian operator and a unitary operator representing time translations. Constructive quantum field theorists have taken up the project of finding such representations. Due to the formidable mathematical challenges involved, they have yet to find a representation for a

\footnotetext{
${ }^{16}$ This issue is discussed further in Fraser (2006).
} 
physically realistic interaction in four spacetime dimensions. However, this does not mean that we should settle for the renormalized interaction picture representation.

The two methods for generalizing the definition of the Fock representation for a free field evaluated in the ensuing two sections are proposals for finding a suitable Hilbert space representation for an interaction. These methods are instances of the two different approaches to treating interactions in a mathematically well-defined framework that have been developed by mathematical physicists. The method considered in the next section follows the constructive approach of constructing a Hilbert space representation for a particular interacting system. The method discussed in the following section adopts the axiomatic approach of specifying a Hilbert space representation by stipulating formal conditions.

\section{Method \#2: Application of the construction that gen- erates a Fock representation}

A Fock representation for a free system is generated from the classical free field by a quantization procedure. One approach to generalizing the definition of a Fock representation to interacting fields is to apply the same mathematical construction to a classical interacting field. The mathematical construction of a Fock representation proceeds by Fourier decomposing the free field satisfying the classical field equation into positive and negative frequency parts and then promoting the coefficients in the decomposition to operators. The proposal is that a $\Phi O K_{1}$ representation is the Hilbert space representation produced when this mathematical construction is applied to an interacting field. This approach seems to be the most promising one for obtaining a Hilbert space representation that supports a quanta interpretation: intuitively, an annihilation operator annihilates a quantum because it is part of a negative frequency solution to the free field equation and a creation operator creates a quantum because it is part of a positive frequency solution to the free field equation.

For the sake of definiteness and simplicity, consider a free bosonic neutral scalar field with 
$m>0, \psi(x) .{ }^{17} \psi(x)$ is a classical field satisfying the classical Klein-Gordon equation

$$
\left(\square+m^{2}\right) \psi(x)=0
$$

The first step in the Fock space construction is to introduce the Fourier decomposition of $\psi(x)$

$$
\begin{gathered}
\psi(x)=\int d^{4} k\left(a^{+}(k) e^{i k \cdot x}+a^{-}(-k) e^{-i k \cdot x}\right) \\
\text { where } k \text { is a four-vector, } \\
\qquad a^{+}(k)=0 \text { if } k_{0}<0 \text { and } \\
a^{-}(k)=0 \text { if } k_{0}>0\left(\text { so } a^{-}(-k)=0 \text { if } k_{0}<0\right)
\end{gathered}
$$

Since we are using the convention for the metric tensor $g_{\mu \nu}$ that $g_{00}=+1, g_{11}=g_{22}=g_{33}=-1$, $e^{i k x}$ and $e^{-i k x}$ are, respectively, positive and negative frequency plane wave solutions ${ }^{18}$ of the classical Klein-Gordon wave equation (Equation (13)); thus, the first term of Equation (14) represents the positive frequency part of $\psi(x)$ and the second term the negative frequency part. $\psi(x)$ satisfies the Klein-Gordon equation; it follows from plugging $\psi(x)$ into Equation (13) that $a^{+}(k)=0$ and $a^{-}(-k)=0$ when $k^{2} \neq m^{2}$. That is, the coefficients are only non-zero when $k_{0}^{2}-\mathbf{k}^{2}=m^{2}$, the relativistic constraint for a single non-interacting particle with rest mass $m$. This relation also implies that, when $a^{+}(k), a^{-}(k)$ are non-zero, $k$ is timelike: $k^{2}=m^{2}>0$. The fact that $k$ is timelike guarantees that the positive-negative frequency decomposition is Lorentz covariant (see Schwinger (1949) for a proof); $a^{+}(k), a^{-}(k)$ are not dependent on the inertial reference frame in which the decomposition is carried out. This means that $a^{+}(k), a^{-}(k)$ are candidates for being physical scalar fields. Furthermore, when $a^{+}(k)$ and $a^{-}(k)$ are promoted to operators, the field operators $\hat{a}^{+}(k)$ and $\hat{a}^{-}(k)$ are relativistically covariant.

Since $\hat{\psi}(x)$ is Hermitian, $\hat{a}^{+}(k)=\left(\hat{a}^{-}(-k)\right)^{\dagger}$. Setting $\hat{a}(k)=\hat{a}^{-}(-k)$ then gives the four-

\footnotetext{
${ }^{17}$ The following is largely based on Roman (1969, pp. 48-52, 79-97, 119).

${ }^{18}$ Plane wave solutions are not normalizable on infinite four-dimensional Minkowski spacetime (see footnote 4). This does not affect the point made here. For a version of the Fock space construction that does not make this assumption see $\S 3.2$ of Wald (1994).
} 
dimensional analogue of Equation (2):

$$
\hat{\psi}(x)=\int d^{4} k\left(\hat{a}^{\dagger}(k) e^{i k \cdot x}+\hat{a}(k) e^{-k \cdot x}\right)
$$

In the interacting case, the crucial difference is that, of course, the classical interacting field $\varphi(x)$ no longer obeys the homogeneous Klein-Gordon equation. It might, for example, obey the following field equation derived from a Lagrangian with a $\varphi(x)^{4}$ self-interaction term

$$
\left(\square+m^{2}\right) \varphi(x)=-4 \lambda \varphi(x)^{3}
$$

The Fourier decomposition for $\varphi(x)$ is

$$
\begin{gathered}
\varphi(x)=\int d^{4} k\left(b^{+}(k) e^{i k \cdot x}+b^{-}(-k) e^{-i k \cdot x}\right) \\
\text { where } k \text { is a four-vector, } \\
b^{+}(k)=0 \text { if } k_{0}<0 \text { and } \\
b^{-}(k)=0 \text { if } k_{0}>0
\end{gathered}
$$

It is possible to carry out this Fourier decomposition; however, plugging $\varphi(x)$ into the interacting field equation does not yield the constraint $k^{2}=m^{2}$. The consequence is that, unlike the free field case, $k$ will, in general, ${ }^{19}$ not be timelike: $k^{2} \neq m^{2}$, so there is no guarantee that $k^{2}>0$. As a result, the decomposition in terms of functions $b^{+}(k), b^{-}(k)$ is typically not covariant (Roman 1969, p. 119). Furthermore, if $b^{+}(k), b^{-}(k)$ were promoted to field operators, they would also fail to be covariant in general; the field operators $\hat{b}^{+}(k), \hat{b}^{-}(k)$ would be inertial reference frame dependent, and therefore not candidates for physical fields.

This is a fatal flaw in the strategy of using the Fourier decomposition of an interacting field to obtain a $\Phi O K_{1}$ representation for it. A fortiori, this procedure does not yield a quanta

\footnotetext{
${ }^{19}$ This is a very minor qualification: for very special interacting field equations, it may happen to be the case that $k^{2}>0$.
} 
interpretation for an interacting system. ${ }^{20}$

The failure of Lorentz covariance furnishes sufficient reason to reject the $\Phi O K_{1}$ representation for an interacting field; however, for the sake of completeness, it is necessary to investigate another obstacle to obtaining a quanta interpretation for this representation. In a nutshell, the other problem is that there are special circumstances which make Fourier analysis an appropriate technique to employ in the free case, but not the interacting case. This problem is unrelated to relativistic considerations. Recall that, in the free case, the definitive argument for regarding states of the form $\hat{a}^{\dagger}(k)|0\rangle$ as states in which a single quantum is present is that there is a correlation between the energies of these states and the energies of the corresponding solutions to the classical field equation. Formally, the mapping $K: S \rightarrow \mathcal{H}$ from the space of positive frequency solutions of the classical field equation $S$ to states in the one-particle Hilbert space $\mathcal{H}$ can be set up using the Fourier decomposition. $K$ is specified by setting $e^{i k \cdot x} \rightarrow \hat{a}^{\dagger}(k)|0\rangle$ when $k_{0}>0$ and $k^{2}=m^{2}$ and $e^{i k \cdot x} \rightarrow 0$ when $k_{0}<0$ and $k^{2}=m^{2}$. This stipulation fully specifies $K$ because any solution of the classical Klein-Gordon equation can be Fourier analyzed in terms of $e^{i k \cdot x}$ with $k^{2}=m^{2}$. It is a contingent fact that functions of the form $e^{i k \cdot x}\left(k^{2}=m^{2}\right)$ are themselves solutions of the Klein-Gordon equation; this happy accident enables us to describe the component $S$ of the mapping $K$ in terms of solutions to the Klein-Gordon equation as well as in terms of Fourier modes $e^{i k \cdot x}\left(k^{2}=m^{2}\right)$.

Consider what happens when one tries to define an analogous mapping $K^{\prime}$ for an interacting field $\hat{\varphi}(x)$. Since we know that, in general, the Fourier decomposition yields operators which are not Lorentz covariant, restrict attention to a single inertial frame of reference. The Fourier decomposition gives the following mapping: $K^{\prime}: X \rightarrow \mathcal{H}_{I}$ is fully specified by setting $e^{i k \cdot x} \rightarrow$ $\hat{b}^{+}(k)|0\rangle$ where $k_{0}>0$ and $k$ satisfies the constraint obtained by substituting the expression for $\varphi(x)$ given by the Fourier decomposition into the interacting field equation and determining the

\footnotetext{
${ }^{20}$ Since Fleming (2001) argues for a reference frame dependent notion of localized quanta for a free system, it is possible that he may also be willing to accept reference frame dependent annihilation and creation field operators for an interacting system. However, ultimately, this concession does not help because such a proposal would still face the additional obstacles outlined in the following paragraphs.
} 
values of $k$ for which $b^{+}(k), b^{-}(k)$ are non-zero; ${ }^{21}$ again, Fourier analysis guarantees that this fully specifies $K^{\prime}$. In this case, functions of the form $e^{i k \cdot x}\left(k_{0}>0\right.$ and $k$ satisfies the constraint) are not solutions of the interacting field equation. Consequently, $X$ is not the set of positive frequency complex solutions to the classical interacting field equation. Fourier analysis guarantees that $X$ contains the set of positive frequency complex solutions, but in addition $X$ also contains other elements. One way of proceeding is to isolate the subset $\bar{S} \subset X$ that contains the positive frequency complex solutions and define the partial mapping $\bar{K}: \bar{S} \rightarrow \mathcal{H}_{I}$. Assuming that the vector addition operation is chosen to be arithmetic addition, $\bar{S}$ is not a vector space because it is not closed under this operation: the interacting field equation is non-linear so the sum of two solutions will in general not be a solution. Since $\bar{S}$ is not a vector space, it cannot be taken to be the vector space for the Hilbert space $\mathcal{H}_{I}$. This is a problem because the specification of $\mathcal{H}_{I}$ is an essential component of the 'Fock' quantization.

To get around this difficulty, employ the original mapping $K^{\prime}: X \rightarrow \mathcal{H}_{I}$, which contains the vector space $X$, for the purpose of the quantization, but appeal to the partial mapping $\bar{K}: \bar{S} \rightarrow \mathcal{H}_{I}$ to justify the interpretation of $\mathcal{H}_{I}$ as the one-particle Hilbert space containing oneparticle states. The interpretation of $\mathcal{H}_{I}$ is secured by establishing a correspondence between the energies of classical solutions and quantum states in $\mathcal{H}_{I}$. But there is at least one serious problem with this maneuver. In the free case, $K$ maps positive frequency complex solutions of the Klein-Gordon equation of the form $e^{i k \cdot x}$ to Hilbert space states of the form $\hat{a}^{\dagger}(k)|0\rangle$. In the interacting case, $\bar{K}$ maps an integral over $e^{i k \cdot x}$ to an element of $X$. In the free case, the imposition of finite volume or periodic volume boundary conditions allows the integral to be replaced by an infinite sum. This may not hold in the interacting case, but assume for the moment that it does (if not, then this could introduce the additional complication of the Hilbert space associated with $X$ being nonseparable). Then $\bar{K}$ maps an infinite sum over $e^{i k \cdot x}$ to a superposition $b^{+}\left(k_{1}\right) \hat{b}^{+}\left(k_{1}\right)|0\rangle+b^{+}\left(k_{2}\right) \hat{b}^{+}\left(k_{2}\right)|0\rangle+\ldots$. There are two problems with this. First, following the same logic as in the free case, if anything should be identified as a one-particle state

\footnotetext{
${ }^{21}$ It is not necessary to use the Fourier decomposition to determine this constraint; the Fourier transform $\varphi(x)=\int d^{4} k b(k) e^{i k x}$ is sufficient for this purpose.
} 
in $\mathcal{H}_{I}$, it is not a state like $\hat{b}^{+}\left(k_{1}\right)|0\rangle$, but a superposition of such states, $\hat{b}^{+}\left(k_{1}\right)|0\rangle+\hat{b}^{+}\left(k_{2}\right)|0\rangle+\ldots$. This confounds the usual quanta interpretation of a Fock representation. Second, there is no reason to expect that the set of superpositions given by $\bar{K}$ contains a set of pairwise orthogonal vectors that spans the Hilbert space because the coefficients $b^{+}\left(k_{i}\right)$ and the $k_{i}$ 's are fixed by the interacting field equation. This line of argument supplies the justification for Redhead's assertion that, for interacting fields, "the equations of motion are in general non-linear so unlike the free-field case we cannot reduce the problem to independent harmonic oscillators (the normal modes)" (1988, p. 20).

In response to the failure of the method of Fourier decomposing an interacting field to yield a quanta interpretation, one might consider generalizing the construction. Instead of Fourier decomposing the classical interacting field into functions of the form $e^{i k \cdot x}$, one might attempt to decompose it into functions of some other form. A suggestion along these lines is mooted in Huggett and Weingard (1994) and Huggett (2000). Huggett floats-but ultimately rejects ${ }^{22}-$ the possibility of extending the oscillator analogy to the interacting case in the following way: "[f]or an interacting field the oscillators do not move independently, but as if they were interconnected: there might be further springs, one between any pair of bobs" (p. 628). Translated into the terms of the present discussion, the suggestion is that instead of decomposing the field into independent oscillators-the plane waves $e^{i k \cdot x}$-the field should be decomposed into coupled oscillators, which are represented by functions of some other form. Huggett and Weingard suspect that this is not possible (p. 376). This is a reasonable conjecture because the proposal faces significant obstacles from two sources. First, there is no guarantee that a function can be decomposed using an arbitrary set of functions; the set of functions of the form $e^{i k \cdot x}$ is special in this respect. Second, even if a workable alternative to Fourier analysis were identified, this resulting decomposition might very well fail to be Lorentz covariant. Since these challenges are both substantial, it seems safe to conclude that it is not possible to obtain an analogue of the Fock representation suited

\footnotetext{
${ }^{22} \mathrm{He}$ argues that, in any case, "if the bobs do not move as SHO's but in some more complex way then there are no SHO's and by analogy no quanta in the field" (p. 628). He may have in mind the point made above that the relativistic energy constraint $k^{2}=m^{2}$ only holds for the Klein-Gordon equation, which can be interpreted as describing an infinite collection of SHO's.
} 
to an interacting field by applying an analogue of the mathematical construction that produces the Fock representation for a free field.

\section{Method \#3: Stipulation of formal conditions}

The method for obtaining a $\Phi O K_{1}$ representation pursued in the previous section was to quantize a given classical interacting field in the same manner in which a given classical free field is quantized to produce a Fock representation. Following the failure of this method, a different method for extending the definition of "Fock representation" to interactions will be pursued in this section. The strategy is to arrive at a Hilbert space representation of the ETCCR's by stipulating formal conditions on the field operators rather than by quantizing a classical field. This strategy is characteristic of the axiomatic approach to QFT. The definition of $\Phi O K_{2}$ representation set out below was first proposed by Wightman, a leading practitioner of axiomatic QFT, and his collaborators. The focus is on the product of the quantization process for a free field instead of the quantization process itself; the formal conditions are gleaned from the properties of a Fock representation listed in Section 2. The hope is that while the quantization procedure for a free field does not produce the desired result when applied to an interacting field, the formal product of the quantization procedure for free systems can be extended to interacting systems and possesses the appropriate features. As we shall see, this wish is not fulfilled.

In order to properly define a $\Phi O K_{2}$ representation, the loose characterizations of operators employed to this point must be refined. In order to be well-defined as operator-valued distributions, the fields must be smeared with test functions. Let $\mathcal{T}$ be a real vector space with scalar product $(f, g)$ and norm $\|f\|=(f, f)^{\frac{1}{2}}$ that serves as the test function space; then, for example, $\phi(f, t)=\int \phi(\mathbf{x}, t) f(\mathbf{x}) d^{3} x$ for $f \in \mathcal{T}$.

Let $\phi(f, t)$ satisfy some quantum field equation ${ }^{23}$ and let $\pi(f, t)$ be its conjugate momentum

\footnotetext{
${ }^{23}$ Specification of a field equation may not be sufficient to isolate a representation that is unique up to unitary equivalence in all cases, but this potential problem is unrelated to the present discussion. See Baez \& Zhou (1992).
} 
field. The definition of a $\Phi O K_{2}$ representation of the ETCCR's ${ }^{24}$ for $\phi(f, t), \pi(f, t)$ has two parts (Gårding \& Wightman 1954; Wightman \& Schweber 1955):

Condition 1. Define, for any $f \in \mathcal{T}$, the operator

$$
c(f, t)=\frac{1}{\sqrt{2}}[\phi(f, t)+i \pi(f, t)]
$$

and define $c^{\dagger}(f, t)$ as the adjoint of $c(f, t)$.

Condition 2. The $\Phi O K_{2}$ representation is the irreducible representation of the ETCCR's for which there exists a normalizable vector (the "no-particle state") $|0\rangle$ such that ${ }^{25}$

$$
c(f, t)|0\rangle=0 \text { for all } f \in \mathcal{T}
$$

These conditions are familiar from the discussion of the Fock representation for a free field in Section 2. This definition is clearly not restricted to free fields; $\phi(f, t)$ may satisfy any field equation. Wightman and Schweber explicitly state that this is the case $(1955$, p. 816$) .{ }^{26}$ Unlike the operators $\hat{b}(k), \hat{b}^{\dagger}(k)$, which were defined using the Fourier decomposition of the classical field, the operators $c(f, t), c^{\dagger}(f, t)$ are Poincaré covariant since $\phi(f, t), \pi(f, t)$ are Poincaré covariant. ${ }^{27}$ These two conditions pick out a representation of the ETCCR's that is unique up to unitary equivalence $^{28}$ (Gårding and Wightman 1954, pp. 624-625; see also Wightman and Schweber 1955,

\footnotetext{
${ }^{24}$ There is an analogous definition of $\Phi O K$ representation for the ETCAR's (equal-time canonical anticommutation relations) (Wightman \& Schweber 1955, pp. 816-820).

${ }^{25}$ The cyclicity of $|0\rangle$ with respect to $c^{\dagger}(f, t)$ follows from the assumption that the Weyl representation of the ETCCR's for $\phi(f, t), \pi(f, t)$ is irreducible, which implies that every non-zero vector in the representation is cyclic with respect to $e^{i \phi(f, t)}, e^{i \pi(f, t)}($ Emch 1972, p. 84).

${ }^{26}$ They use the terminology of "Heisenberg or interaction representation quanta." In the interaction representation the field obeys the free Klein-Gordon equation and in the Heisenberg representation the field evolves with the full interaction Hamiltonian.

${ }^{27}$ Unlike $\hat{b}(k), \hat{b}^{\dagger}(k)$, the operators $c(k), c^{\dagger}(k)$ do not arise from coefficients in the Fourier decomposition of the classical interacting field.

${ }^{28}$ Gårding and Wightman's proof establishes that, if the representation exists, then it is unique up to unitary equivalence, but it is not clear that it establishes that the representation necessarily exists for an interacting field (i.e., that there exists a representation containing a normalizable $|0\rangle$ ). I will assume that such a representation exists and argue that, even if the representation does exist, it cannot sustain a quanta interpretation.
} 
pp. 819-822). (Of course, while the $\Phi O K_{2}$ representation for a field $\phi(f, t)$ satisfying a specified field equation is unique up to unitary equivalence, the $\Phi O K_{2}$ representations for fields $\phi(f, t)$ and $\phi^{\prime}(f, t)$ satisfying different field equations may not be unitarily equivalent.) Since the Fock representation for a free field satisfies these two conditions (see Section 2), when $\phi(f, t)$ satisfies a Klein-Gordon equation, the $\Phi O K_{2}$ representation coincides with the Fock representation.

A $\Phi O K_{2}$ representation also shares some of the other properties of a Fock representation rehearsed in Section 2. It follows from the definitions in Condition 1 and the fact that $\phi(f, t)$, $\pi(f, t)$ satisfy the Weyl form of the ETCCR's that $c(f, t), c^{\dagger}(f, t)$ satisfy the usual ETCCR's ${ }^{29}$

$$
\begin{gathered}
{[c(f, t), c(g, t)]=0=\left[c^{\dagger}(f, t), c^{\dagger}(g, t)\right]} \\
{\left[c(f, t), c^{\dagger}(g, t)\right]=(f, g)}
\end{gathered}
$$

Most significant for our purposes is the fact that a $\Phi O K_{2}$ representation has a well-defined total number operator $N(t)=\sum_{j=1}^{\infty} c^{\dagger}\left(f_{j}, t\right) c\left(f_{j}, t\right)$ where $f_{j}$ are members of any orthonormal set in $\mathcal{T}$ (Wightman \& Schweber 1955, p. 822; Gårding \& Wightman 1954, p. 625; Wightman 1967b, p. 188). Moreover, any representation in which $N(t)$ is well-defined (where $c^{\dagger}\left(f_{j}, t\right), c\left(f_{j}, t\right)$ are defined as in Condition 1) is a direct sum of representations in which both of the conditions

${ }^{29}$ Consider the case in which $\phi(f, t)$ is a free field. Note that Wightman's definitions imply

$$
\begin{aligned}
\phi(\mathbf{x}, t) & =\int \frac{d^{3} k}{(2 \pi)^{\frac{3}{2}} \sqrt{2}}\left[c^{\dagger}(\mathbf{k}, t) e^{i k \cdot x}+c(\mathbf{k}, t) e^{-i k \cdot x}\right] \\
\pi(\mathbf{x}, t) & =\int \frac{i d^{3} k}{(2 \pi)^{\frac{3}{2}} \sqrt{2}}\left[c^{\dagger}(\mathbf{k}, t) e^{i k \cdot x}-c(\mathbf{k}, t) e^{-i k \cdot x}\right]
\end{aligned}
$$

The first expression differs from (2) in Section 2 by a factor of $\omega_{\mathbf{k}}^{-\frac{1}{2}}$ inside the integral and the second differs from (3) by a factor of $\omega_{\mathbf{k}}^{\frac{1}{2}}$ inside the integral. These factors cancel when $\phi(\mathbf{x}, t), \pi(\mathbf{x}, t)$ are multiplied, so Wightman's $\phi(f, t), \pi(f, t)$ obey the same ETCCR's as their counterparts in section 2 . In $(\mathbf{k}, t)$-space

$$
\begin{gathered}
{\left[c\left(\mathbf{k}_{1}, t\right), c\left(\mathbf{k}_{2}, t\right)\right]=0=\left[c^{\dagger}\left(\mathbf{k}_{1}, t\right), c^{\dagger}\left(\mathbf{k}_{2}, t\right)\right]} \\
{\left[c\left(\mathbf{k}_{1}, t\right), c^{\dagger}\left(\mathbf{k}_{2}, t\right)\right]=\omega_{\mathbf{k}} \delta\left(\mathbf{k}_{1}-\mathbf{k}_{2}\right)}
\end{gathered}
$$

which differs from the ETCCR's in Section 2 by a factor of $\omega_{\mathbf{k}}$.

Note also that, if $\phi(f, t), \pi(f, t)$ are regarded as fields at arbitrary times rather than a fixed time, $\pi(f, t) \neq$ $\frac{d \phi^{\prime}(f, t)}{d t} ; \pi(f, t)=\frac{d \phi^{\prime}(f, t)}{d t}$ where $\phi^{\prime}(\mathbf{k}, t)=\frac{1}{\omega_{\mathbf{k}}} \phi(\mathbf{k}, t)$. 
hold (Dell'Antonio, Doplicher \& Ruelle 1966) ${ }^{30}$. This result provides assurance that the $\Phi O K_{2}$ representation is the unique generalization of a Fock representation capable of furnishing a quanta interpretation: imposing the definition of $c^{\dagger}(f, t), c(f, t)$ set out in Condition 1 and the condition that $N(t)$ be well-defined also yields a direct sum of $\Phi O K_{2}$ representations. ${ }^{31}$

A $\Phi O K_{2}$ representation for an interacting field $\phi(f, t)$ possesses a total number operator but, once again, this is an insufficient basis for an interpretation in terms of particlelike entities. In addition, it is necessary to establish that each of the eigenstates of $N$ is a state in which the system possesses the appropriate physical properties for a state in which the indicated number of quanta is present. It turns out that this cannot be done if $\phi(f, t)$ is an interacting field. Recall from the discussion in Section 2 that, in order for the no-particle state to have the correct energy for a state in which no quanta are present and to possess the expected property of invariance under Poincaré transformations, the no-particle state must coincide with the vacuum. One would expect a state in which no quanta are present to exhibit these properties regardless of whether the system is free or interacting. If, in addition to the two conditions stated above, it is assumed that the no-particle state in a $\Phi O K_{2}$ representation is the vacuum state, then it can be proven that $\phi(f, t)$ cannot be a non-trivial interacting field (Emch 1972, p. 242). Essentially, ${ }^{32}$ this result is proven by calculating the vacuum expectation values of products of the fields. The vacuum expectation value of any product of $n$ (where $n>2$ ) fields can be expressed solely in terms of the vacuum expectation values of products of two fields. This implies that the $S$-matrix is the identity (Garczyński 1964). That is, no interaction occurs; the initial state of the system

\footnotetext{
${ }^{30}$ Chaiken (1968) proves the same result for an $N$ specified using the exponentiated form of the ETCCR $N a^{*}(\varphi)=a^{*}(\varphi)(N+1)$ (where $a^{*}(\varphi)$ is the creation operator for the wavefunction $\varphi$ ) when the spectrum of $N$ is bounded from below (1.3 Theorem, p. 167). (cf. Halvorson and Clifton (2002, p. 203 n. 5)).

${ }^{31} \mathrm{It}$ is worth pointing out the differences between this definition of a $\Phi O K_{2}$ representation and another rigorous generalization of the definition of Fock representation that has recently been discussed by philosophers (see, e.g., Arageorgis et. al. (2002)), even though the alternative definition is inapplicable to interactions. Kay (1978) sets out a definition of Fock representation that is suitable for arbitrary globally hyperbolic spacetimes, but is only applicable to free fields. In a stationary spacetime, this definition picks out a representation that is unique up to unitary equivalence. Briefly, for free fields on Minkowski spacetime, the main differences between Kay's definition and the definition of a $\Phi O K_{2}$ representation are that (1) while Kay's definition requires that the no-particle state coincide with the physical vacuum, this may not be true in a $\Phi O K_{2}$ representation (see below) and (2) Condition 1 for a $\Phi O K_{2}$ representation is not a part of Kay's definition.

${ }^{32}$ Emch states and proves this result within the algebraic framework for QFT. For a brief overview of the relevant features of the algebraic approach, see the Appendix to Earman and Fraser (2006).
} 
is identical to the final state. This result entails that, in the presence of a non-trivial interaction, the no-particle state is not the vacuum state. Therefore, a $\Phi O K_{2}$ representation does not furnish a quanta interpretation for an interacting system.

To see that a $\Phi O K_{2}$ representation for an interacting field also fails to ascribe the correct properties to higher particle states, consider the example of a Lagrangian with a $\phi^{4}$ interaction term. The following is the formal expression for the Hamiltonian $H^{\phi^{4}}$ in terms of $c^{\dagger}(\mathbf{k}, t)$, $c(\mathbf{k}, t)$, the 'unsmeared' Fourier transforms of the annihilation and creation operators defined in Condition 1 (where : : represents normal ordering):

$$
\begin{aligned}
& H^{\phi^{4}}=\int d k c^{\dagger}(\mathbf{k}, t) c(\mathbf{k}, t)+ \\
& \lambda \iiint \int d k d k^{\prime} d k^{\prime \prime} d k^{\prime \prime \prime} \frac{\delta\left(\mathbf{k}+\mathbf{k}^{\prime}+\mathbf{k}^{\prime \prime}+\mathbf{k}^{\prime \prime \prime}\right)}{4}: \\
& \quad\left[c^{\dagger}(\mathbf{k}, t)+c(\mathbf{k}, t)\right] \cdots \\
& {\left[c^{\dagger}\left(\mathbf{k}^{\prime \prime \prime}, t\right)+c\left(\mathbf{k}^{\prime \prime \prime}, t\right)\right]:}
\end{aligned}
$$

It can be confirmed that the no-particle state $|0\rangle$ does not coincide with the vacuum: the interaction term contains one term with four creation operators; as no other term in $H^{\phi^{4}}$ contains four creation operators, this term is not offset by any other term, which means that $H^{\phi^{4}}|0\rangle \neq 0$. Furthermore, $|0\rangle$ is not an eigenstate of $H^{\phi^{4}}$, so it is not possible to make $|0\rangle$ coincide with the vacuum by adding a finite constant to $H^{\phi^{4}}$.

The result of applying $H^{\phi^{4}}$ to a one-particle state is the following:

$$
H^{\phi^{4}} c^{\dagger}(\mathbf{k}, t)|0\rangle=\sqrt{\mathbf{k}^{2}+m^{2}} c^{\dagger}(\mathbf{k}, t)|0\rangle+\ldots
$$

where the ellipsis stands in for the terms resulting from application of the interaction term of $H^{\phi^{4}}$. Clearly, $c^{\dagger}(\mathbf{k}, t)|0\rangle$ is not an eigenstate of $H^{\phi^{4}}$. On many interpretations of quantum theory, this means that $c^{\dagger}(\mathbf{k}, t)|0\rangle$ is a state which does not possess a definite value of energy. This seems strange for a state which is supposed to definitely contain a single quantum. However, set this issue aside, because there is a serious problem with interpreting $c^{\dagger}(\mathbf{k}, t)|0\rangle$ as a state in which a 
single quantum is present that is independent of controversies surrounding the interpretation of quantum theory. This argument takes the form of a dilemma. There are two positions that could be taken on the correct relativistic energy possessed by a single quantum associated with the interacting field $\phi(x)$ : either that it should be $\sqrt{\mathbf{k}^{2}+m^{2}}$, the relativistic energy for a single noninteracting particle, or that it should not be $\sqrt{\mathbf{k}^{2}+m^{2}}$ because the field equation for $\phi(x)$ contains a self-interaction term. If the former position is taken, then $c^{\dagger}(\mathbf{k}, t)|0\rangle$ cannot be interpreted as a state in which one quantum is present because the expectation value for energy in this state is not $\sqrt{\mathbf{k}^{2}+m^{2}}$. If the latter position is adopted, we are left without any guidance from special relativity about what the correct energy for a single quantum state should be; consequently, we have no grounds for claiming that $c^{\dagger}(\mathbf{k}, t)|0\rangle$ is a single quantum state. Therefore, $c^{\dagger}(\mathbf{k}, t)|0\rangle$ cannot be interpreted as a state in which one quantum is present.

In sum, a $\Phi O K_{2}$ representation does not sustain a quanta interpretation for any interacting field because the no-particle state is not the vacuum state. In addition, there are no grounds for interpreting one-particle states of the form $c^{\dagger}(\mathbf{k}, t)|0\rangle$ as states in which one quantum is present.

In response to the $\Phi O K_{2}$ representation's failure to support a quanta interpretation for an interacting system, one might want to further generalize the definition of Fock representation. A prerequisite for a quanta interpretation is that a total number operator $N(t)=$ $\sum_{j=1}^{\infty} c^{\dagger}\left(f_{j}, t\right) c\left(f_{j}, t\right)$ exist, but there is no reason that $c^{\dagger}(f, t), c(f, t)$ must be defined as in Condition 1. Further motivation for this move is that, in a Fock representation for a free field, Condition 1 is a product of the Fourier decomposition procedure for quantizing a classical free field. However, this proposal requires fleshing out: clearly, in the absence of a definition of $c^{\dagger}(f, t)$, $c(f, t)$ in terms of $\phi(f, t), \pi(f, t)$, the requirement that a total number operator exist or that the no-particle state exists is insufficient to pick out a representation of the ETCCR that is unique up to unitary equivalence. But how should $c^{\dagger}(f, t), c(f, t)$ be defined in terms of $\phi(f, t), \pi(f, t)$ in the presence of an interaction? A well-motivated answer to this question would require revisiting the issue of how to quantize an interacting field. Once again, what is needed is an analogue of the Fourier decomposition of a classical free field that is appropriate for an classical interacting 
field and, as argued in the previous section, it is overwhelmingly unlikely that such a procedure could be found. The discussion in this section has raised another potential difficulty: it is not automatic that the one-particle states will (or even should!) possess the energies $\sqrt{\mathbf{k}^{2}+m^{2}}$. If not, then most compelling argument for interpreting one-particle states as states in which one quantum is present is undermined.

\section{Scattering theory does not support a quanta interpre- tation}

To recapitulate the argument thus far, we set out to find a mathematical representation for an interacting system that sustains a quanta interpretation in the same manner that a Fock representation furnishes a quanta interpretation for a free system. All three methods for accomplishing this have proven unsuccessful. Generalizations of two of the strategies cannot be ruled out entirely, but success seems exceedingly unlikely. Since in QFT there is no known alternative for establishing that a free or interacting system exhibits particlelike properties, this would seem to be a fatal blow to the project of interpreting any realistic physical system-which is bound to interact-in terms of particlelike entities.

In a last-ditch attempt to save the quanta interpretation of QFT, one could try to find a convincing argument that the quanta interpretation for free systems is all that is needed to interpret an interacting system in terms of quanta. This position can be motivated by considering how particle physicists interpret a theory in terms of particlelike entities. For experimentalists, it is immaterial whether or not an interacting system can be given a quanta interpretation; this question is irrelevant because the only data collected from scattering experiments is for systems with negligibly small interactions. Roughly speaking, this is because the data is collected long before and long after the collision between the 'particles' occurs. The input of the experiment is the prepared system of particlelike entities of certain types with certain properties; this system is 
prepared in such a way that interactions are negligible ${ }^{33}$ (e.g., the particlelike entities are very far apart). ${ }^{34}$ Similarly, the output of the experiment is the measured system of particlelike entities of certain (possibly different) types with different properties; measurements are performed on the system at a time when interactions are negligible. These conditions are reflected in scattering theory: ${ }^{35}$ it is assumed that the interacting system tends to a free system at asymptotically early and late times (i.e., as the time variable $t$ tends to plus or minus infinity). At these times, the free system can be given a Fock representation and a quanta interpretation in the usual way.

Bain has advanced a quanta interpretation for QFT based on scattering theory. He proposes that "a 'particle' be considered a system that minimally possesses an asymptotic state (i.e., a system that is free for all practical purposes at asymptotic times)" (Bain 2000, p. 394). Since the asymptotic state is a free state, it possesses a Fock representation and can be given an interpretation in terms of quanta. Bain urges that this is sufficient grounds for interpreting interacting states at intervening times as states in which quanta are present. Teller opposes this position, arguing that such a quanta interpretation is "severely limited" because the total number operator $N_{\text {free }}$ defined in the Fock representation at asymptotic times does not exist at intervening finite times (1995, p. 123). Bain counters that

a particle interpretation should not be dependent on the existence of a (free field) [total] number operator. To require otherwise seems to me to be placing undue emphasis on the free theory. ... Whether or not [a system that possesses an asymptotic state] has a corresponding [total] number operator, I would claim, is irrelevant. (Bain 2000, p. 394)

The analysis in the preceding sections provides a basis for settling this dispute about whether the existence of Fock representations for asymptotic free systems is a sufficient basis on which

\footnotetext{
${ }^{33}$ Set aside the issue of whether the asymptotic system is genuinely free or only free to a very good approximation. If the latter, then it cannot be given a quanta interpretation for all the reasons stated in the preceding sections.

${ }^{34}$ Exclude quantum chromodynamics from consideration because, due to confinement, interacting systems approach free systems in the limit of small distance rather than large distance. See Redhead (1988, p. 21).

${ }^{35}$ For example, LSZ or Haag-Ruelle scattering theory. For a discussion of the latter see Earman and Fraser (2006, Section 6).
} 
to regard quanta as fundamental entities in our ontology. The evidence weighs against Bain's definition of 'particle'. The underlying problem is a weakness in Bain's approach to ontology. The point at issue is whether entities with certain properties-particlelike properties-exist. More precisely, does QFT support the inclusion of particlelike entities as fundamental entities in our ontology? Bain contends that the fact that there is no free field total number operator $N_{\text {free }}$ at intervening times is irrelevant. But, since the question is whether there are entities with particlelike properties at finite times, what is certainly relevant is whether the interacting system contains entities that are countable. In terms of the formalism, it is relevant whether a surrogate for $N_{\text {free }}$ is available at finite times. Bain does not offer any substitute for $N_{\text {free }}$; he does not point to any evidence for the existence of particlelike entities in the presence of an interaction (e.g., that states of an interacting system possess the expected energies for states in which a definite number of quanta are present). Instead, Bain redefines 'quanta' to fit the evidence that QFT does provide. But lack of evidence is not the sort of thing that can be defined away in the realm of ontology. The 'quanta' in Bain's scheme may not possess any particlelike properties in the presence of interactions. Appealing to the theory of a free system with which the interacting system is associated in an idealized infinite past or future does not fill this gap in the evidence.

The investigation conducted in the preceding sections establishes that the evidence that Bain omits in fact cannot be supplied. It is not possible to produce a mathematical representation for an interacting system that is relevantly similar to the Fock representation for a free system. No substitute for $N_{\text {free }}$ is available; it is not possible to identify $n$-particle states which possess the correct energies. Therefore, we cannot ascribe particlelike properties to a system in a scattering experiment at finite times, and this final attempt to extend the quanta interpretation to interacting systems is also unsuccessful. 


\section{Conclusion}

A Fock representation sustains a quanta interpretation for a free field. The goal of this paper was to determine whether an interacting field possesses a mathematical representation that sustains a quanta interpretation in the same manner. The simplest solution would have been to use the Fock representation for a free field to represent an interacting field. This option was ruled out by Haag's theorem. It then became necessary to find another Hilbert space representation for an interacting field. In principle, there are two ways to generalize the definition of a Fock representation to cover interacting systems, each of which is an instance of one of the two approaches to arriving at a formulation of QFT. The constructive method is to quantize a classical interacting field by carrying out the same mathematical construction that, for a free field, generates a Fock representation. The axiomatic method is to pick out a unique (up to unitary equivalence) Hilbert space representation of the ETCCR by stipulating that it share a set of formal properties with a Fock representation. Neither of these approaches is successful, and the prospects for success of generalizations of these methods are negligibly small. A Hilbert space representation cannot be constructed by Fourier decomposing a classical interacting field because the resulting expressions are not relativistically covariant, and therefore are not candidates for physical fields in relativistic QFT. The $\Phi O K_{2}$ representation for an interacting field that is picked out by formal conditions does not support a quanta interpretation because, for all non-trivial interactions, the no-particle state does not coincide with the vacuum and, typically, the argument that one-particle states have the energy expectation values that special relativity assigns to single particle states is undercut.

These conclusions are significant for metaphysics as well as for the foundations of QFT. The metaphysical implication is that QFT does not support the inclusion of particlelike entities in our ontology. At least on the surface, QFT is a theory of fields. The only known method of interpreting a QFT in terms of particlelike entities is the quanta interpretation that naturally

arises from the Fock representation for a free system. The arguments presented here establish that it is not possible to extend this quanta interpretation to an interacting system. One response 
would be to find another way of interpreting interacting fields in terms of particlelike entities, one that does not require a Fock-type Hilbert space representation. But this is a program, not a solution, and even at that a program without an obvious starting point. Therefore, since in the real world there are always interactions, QFT does not furnish grounds for regarding particlelike entities as constituents of reality.

A second line of response would be to agree that quanta are not part of the ontology of fundamental entities described by QFT, but to argue that nevertheless quanta do exist according to QFT. For example, Wallace considers the 'particle' concept to be an emergent concept (see, e.g., Wallace (2001)). For this to be a viable response, the cogency of the distinction between fundamental and less fundamental entities must be defended and a case must be made for admitting additional, non-fundamental entities into our ontology. Alternatively, the quanta concept may be regarded as a concept that is only approximately or ideally applicable because it is restricted to free systems. In the context of a scattering experiment, free systems occur in the idealized limits of infinitely early and late times. Teller suggests that "we regard this idealization as one of the ways in which the interpretation's similarity relation between the model and the world is only approximate" (1995, p. 124). Again, a case would have to be made that the similarity relation between the model and the world need only be approximate rather than exact. However, the important question-which remains outstanding-is not the status of quanta, but what fundamental entities are allowed into our ontology by QFT.

The reasons that an interacting system cannot be given a quanta interpretation are also illuminating for the foundations of QFT. Scattered remarks in the philosophical literature on QFT often convey the impression that interacting fields do not admit Fock representations due to problems representing two-particle (and three-particle, etc.) states in such a framework (for an explicit example see Bain (2000, p. 393)). One rationale for this assessment is that, for a free system, the energy of a two-particle state is simply the sum of energies of the component one-particle states; however, this is not the case for an interacting system. This property of a free system is built into the definition of a Fock representation: a two-particle state is the 
direct product of component one-particle states. However, the above analysis reveals that this discrepancy is not the primary difficulty. Problems already emerge at the level of no-particle and one-particle states. Furthermore, the source of these problems is the special theory of relativity. Haag's theorem relies on relativistic premises $;{ }^{36}$ the Fourier decomposition is not covariant under Poincaré transformations; the no-particle state in a $\Phi O K_{2}$ representation is not invariant under Poincaré transformations; and special relativity may not supply the correct assignment of energies to one-particle states. For a free system, special relativity and the linear field equation conspire to produce a quanta interpretation. For an interacting system, the combination of special relativity and the nonlinear field equation is not so fortuitous; as a result, there is no quanta interpretation and there are no quanta.

\section{Acknowledgements}

For helpful comments, thanks to an anonymous referee, Gordon Belot, Tony Duncan, Nick Huggett, Wayne Myrvold, Laura Ruetsche, Andrew Wayne, and especially John Earman. I would also like to thank the Social Sciences and Humanities Research Council of Canada for financial support in the form of a Doctoral Fellowship.

\footnotetext{
${ }^{36}$ See Earman and Fraser (2006, Section 4) for details.
} 


\section{References}

Arageorgis, A., Earman, J., \& Ruetsche, L. (2002). Weyling the time away: the non-unitary implementability of quantum field dynamics on curved spacetime. Studies in History and Philosophy of Modern Physics, 33, 151-184.

(2003). Fulling non-uniqueness and the Unruh effect: A primer on some aspects of quantum field theory. Philosophy of Science, 70, 164-202.

Baez, J., \& Zhou, Z (1992). On quantum fields satisfying a given wave equation. Jour. Funct. Analysis, 106, 439-453.

Bain, J. (2000). Against particle/field duality: asymptotic particle states and interpolating fields in interacting QFT (Or: Who's afraid of Haag's theorem?). Erkenntnis, 53, 375-406.

Bogolubov, N. N., Logunov, A. A., \& Todorov, I. T. (1975). Introduction to Axiomatic Quantum Field Theory. Reading, MA: W. A. Benjamin.

Bratteli, O. \& Robinson, D. W. (1996). Operator Algebras and Quantum Statistical Mechanics 2, 2nd ed. Berlin: Springer-Verlag.

Clifton, R., \& Halvorson, H. (2001). Are Rindler quanta real? Inequivalent particle concepts in quantum field theory. Brit. J. Phil. Sci., 52, 417-470.

Chaiken, J. M. (1968). Number operators for representations of the canonical commutation relations. Communications in Mathematical Physics, 8, 164-184.

Dell'Antonio, G. F., Doplicher, S., \& Ruelle, D. (1966). A theorem on canonical commutation and anticommutation relations. Commun. Math. Phys., 2, 223-230.

Earman, J., \& Fraser, D. (2006). Haag's theorem and its implications for quantum field theory. Erkenntnis, 64, 305-344.

Emch, G. (1972). Algebraic Methods in Statistical Mechanics and Quantum Field Theory. New York: Wiley. 
Fleming, G. (2001). Newton-Wigner meets Reeh-Schlieder. Philosophy of Science, 67, S495-515. Fraser, D. (2006). Philosophical implications of the treatment of interactions in quantum field theory. Ph.D. Dissertation. University of Pittsburgh. Available at http://etd.library.pitt.edu/ ETD/available/etd-07042006-134120/.

Garczyński, W. (1964). The necessary and sufficient conditions in terms of Wightman functions for a field to be a generalized free field. Journal of Mathematical Physics, 5, 714-719.

Gårding, L., \& Wightman, A. (1954). Representations of the commutation relations. Proc. Natl. Acad. Sci. U.S., 40, 622 .

Glimm, J. (1969). Models for quantum field theory. In R. Jost (ed.), Local Quantum Field Theory (pp. 97-119). New York: Academic Press.

Glimm, J., \& Jaffe, A. (1969). Infinite renormalization of the Hamiltonian is necessary. Journal of Mathematical Physics, 10, 2213-2214.

— (1970). The $\lambda\left(\phi^{4}\right)_{2}$ quantum field theory without cutoffs: III. The physical vacuum. Acta Mathematica, 125, 203-267.

Haag, R. (1992). Local Quantum Physics. Berlin: Springer-Verlag.

Hall, D., \& Wightman, A. S. (1957). A theorem on invariant analytic functions with applications to relativistic quantum field theory. Det Kongelige Danske Videnskabernes Selskab, Matematiskfysiske Meddelelser, 31, nr 5, 1-41.

Halvorson, H., \& Clifton, R. (2002). No place for particles in relativistic quantum theories? Philosophy of Science, 69, 1-28.

Heathcote, A. (1989). Theory of causality: causality = interaction (as defined by a suitable quantum field theory). Erkenntnis, 31, 77-108.

Huggett, N. (1999). Atomic metaphysics. Journal of Philosophy, 96, 5-24.

(2000). Philosophical foundations of quantum field theory. Brit. J. Phil. Sci., 51, 617637. 
Huggett, N., \& Weingard, R. (1994). Interpretations of quantum field theory. Philosophy of Science, 61, 370-388.

Kay, B. S. (1978). Linear spin-zero quantum fields in external gravitational and scalar fields. Commun. Math. Phys., 62, 55-70.

Lopuszanski, J. (1965). On the unitary inequivalent representations in the quantum field theory and the many body problem. Acta Physica Hungarica, 19, 29-44.

Malament, D. (1996). In defense of dogma: Why there cannot be a relativistic quantum mechanics of (localizable) particles. In R. Clifton (ed.), Perspectives on Quantum Reality (pp. 1-10). Boston: Kluwer.

Redhead, M. (1988). A philosopher looks at quantum field theory. In H. R. Brown and R. Harré (eds.), Philosophical Foundations of Quantum Field Theory (pp. 9-23). New York: Oxford University Press.

Redhead, M., \& Teller, P. (1992). Particle labels and the theory of indistinguishable particles in quantum mechanics. British Journal for Philosophy of Science, 43, 201-218.

Reed, M., \& Simon, B. (1975). Methods of Modern Mathematical Physics II: Fourier Analysis, Self-Adjointness. Academic Press: New York.

Roman, P. (1969). Introduction to Quantum Field Theory. New York: John Wiley and Sons. Ruetsche, L. (2002). Interpreting quantum field theory. Philosophy of Science, 69, 348-378.

Schwinger, J. (1949). Quantum Electrodynamics. II. Vacuum Polarization and Self-Energy. Physical Review, 75, 651-679.

Streater, R. F. (1965). Spontaneous breakdown of symmetry in axiomatic theory. Proceedings of the Royal Society of London, Series A, 287, 510-518.

Streater, R. F., \& Wightman, A. S. (2000). PCT, Spin and Statistics, and All That. New York: W. A. Benjamin. 
Teller, P. (1995). An Interpretative Introduction to Quantum Field Theory. Princeton, NJ: Princeton University Press.

Wald, R. (1994). Quantum Field Theory in Curved Spacetime and Black Hole Thermodynamics. Chicago: University of Chicago Press.

Wallace, D. (2001). The emergence of particles from bosonic quantum field theory. Available at archiv.org/quant-ph/0112149.

Wightman, A. S. (1967a). Introduction to some aspects of the relativistic dynamics of quantized fields. In M. Lévy (ed.), Cargèse Lectures in Theoretical Physics, High Energy Electromagnetic Interactions and Field Theory (pp. 171-291). New York: Gordon and Breach.

— (1967b). Progress in the foundations of quantum field theory. In C. R. Hagen, G. Guralnik, and V. S. Mathur (eds.), Proceedings of the 1967 International Conference on Particles and Fields (pp. 187-218). New York: Interscience Publishers.

Wightman, A. S., \& Schweber, S. (1955). Configuration space methods in relativistic quantum field theory. I. Physical Review, 98, 812-837. 